\title{
Special issue on eMaintenance solutions and technologies
}

\author{
Guest Editorial
}

\author{
Uday Kumar $\cdot$ Aditya Parida $\cdot$ Ramin Karim
}

Published online: 19 April 2011

(C) The Society for Reliability Engineering, Quality and Operations Management (SREQOM), India and The Division of Operation and Maintenance, Lulea University of Technology, Sweden 2011

The term eMaintenance was coined in the early 2000s and has gained its popularity as a component of the eManufacturing and eBusiness concepts, which exploit and gain strength from the emerging information and communication technologies. eMaintenance is a new concept and can be defined as a technique for maintenance support, which includes the resources, services and management necessary to enable and implement a proactive decision making process. This support includes not only eTechnologies, but also eMaintenance activities such as eMonitoring, eDiagnosis, and ePrognosis.

This special issue reflects the challenges and future trends discussed during the First International Congress on eMaintenance Technologies and Solutions "eMaintenance 2010", held in Luleå, June 22-24, 2010. The Congress was initiated and organized by the Division of Operation and Maintenance Engineering of Luleå University of Technology, Luleå, Sweden. The main theme of the congress was "Trends in Technologies and Methodologies, Challenges, Possibilities and Applications of eMaintenance". While most of the articles have been taken from the proceedings of eMaintenance 2010, a few invited articles are also included in this special issue. In total, nine papers have been selected for this issue after peer reviewing of the manuscript.

The first paper, "Maintenance optimization models and criteria", outlines the reasons for the emergence of the eMaintenance concept and reviews the literature on maintenance optimization models, with a specific focus on

U. Kumar $(\bowtie) \cdot$ A. Parida $\cdot$ R. Karim

Division of Operation and Maintenance Engineering,

Luleå University of Technology, Luleå, Sweden

e-mail: Uday.Kumar@1tu.se the optimization criteria and objectives used. To overcome the flaws in the present optimization models, a generic classification framework of maintenance optimization models is presented. The framework is a starting point for the development of business-specific optimization models and enables decision making in eMaintenance.

The authors of the second paper, "Economical and technological prospects for eMaintenance", have elaborated the benefits of eMaintenance and its influence in the life cycle cost of an asset, providing a critical assessment of the current status. The article seeks to assess how eMaintenance end users can benefit from eMaintenance and deals with the financial implications related to its adoption. The main explanation of the benefits is that they result from the new capabilities provided by eMaintenance. If the eTechnologies provide some of these capabilities, maximizing the eMaintenance benefits for the overall maintenance efficiency is possible. However, there are some business and human-related issues that need to be resolved before the actual application of remote maintenance.

The paper, "Organizational dimension of eMaintenance: a contextual perspective", introduces and discusses a contextual perspective for better understanding of the opportunities and challenges associated with organizational learning and innovation in eMaintenance deployment. To achieve success, eMaintenance needs to be aligned with the overall organizational objectives.

Risk management in eMaintenance activities involves a trade-off between protection, on the one hand, and functionality, performance and ease-of-use, on the other.

This aspect is considered in the article "An enterprise risk management framework for evaluation of eMaintenance", where a risk management framework for the evaluation of eMaintenance solutions is proposed. Further, 
it is essential to focus and concentrate on human resource restructuring, maintenance agreements and training. Each maintenance employee (technician, engineer or leader) needs to be capable of dealing with the speed of information flow and understanding the overall structure.

The paper on "The role of information logistics and data warehousing in educational facilities asset management" provides a case study where the complexity of maintenance documents, or data warehousing, and information flow are addressed, among other issues.

eMaintenance is more than the implementation of a maintenance strategy or a maintenance plan. The reasons why the concept of eMaintenance has emerged recently are rooted in the new capabilities provided by the technology: electronics, communication systems and decision support systems.

The paper "A systems approach to integrated eMaintenance of large engineering plants" includes condition monitoring in the maintenance decision process and proposes a maintenance strategy for large plants involving an integration of both time- and condition-based maintenance.

In addition, eMaintenance systems can compare product performance through globally networked monitoring systems to allow companies to focus on degradation monitoring and prognostics rather than fault detection and diagnostics. In line with this, the paper "Application of dynamic benchmarking of rotating machinery for eMaintenance" elaborates the use of data mining of vibration data to obtain the global condition of the plant and the relationship of this parameter with the overall performance. In fact, data mining is one of the most urgent industrial issues for the realization of knowledge-based operation and maintenance of plants. The information flow collected by the eMaintenance platform has to be used for behaviour learning and rule extraction purposes. Hence, a knowledgebased system can be achieved through the intelligent conversion of data into information, and information into knowledge. The paper "Case-based reasoning applied to geometric production measurements" proposes a methodology for quality assurance through measurements from products and the continuous collection of data to allow adjustments in the production line. This intelligent knowledge management is also relevant for maintenance. The paper on "Reliability prediction using support vector regression" shows this conversion of data into real knowledge. A proposal for reliability prediction from stored information for calculation of the remaining useful life of the systems is discussed.

The evolution of eTechnologies plays a crucial role in support of solutions to the challenges of the future and as an aid to management in choosing the right maintenance decision. The rapid development in sensor technology, signal processing and other technologies related to condition monitoring and diagnostics increases the possibilities of utilizing data from multiple origins and sources and data of different types. In addition, by networking remote manufacturing plants, eMaintenance provides a multisource knowledge and data environment. The potential applications in this area include equipment failure prognosis based on the current condition and projected usage, or remaining life prediction for machinery components. In fact, eMaintenance provides companies with predictive intelligence tools to monitor their assets through Internetbased wireless communication systems to prevent them from unexpected breakdown.

Furthermore, eMaintenance can be considered as a major pillar that supports the successful integration of eManufacturing and eBusiness. There are numerous opportunities to respond to the objectives of integration and global optimization of various business processes using eMaintenance solutions. This also provides us with new opportunities for research and development.

It is our sincere hope that this special issue will be highly useful and appreciated by both industry and academia. 